\title{
Chitosan/NaF Particles Prepared Via Ionotropic Gelation: Evaluation of Particles Size and Morphology
}

\author{
Glória Tamiris Farias da Silva Furtado ${ }^{a} \bowtie$, Thiago Bizerra Fideles ${ }^{a}$, Rita de Cassia Alves Leal Cruz ${ }^{a}$, \\ José William de Lima Souza ${ }^{a}$, Miguel Angel Rodriguez Barbero ${ }^{b}$, Marcus Vinicius Lia Fook ${ }^{a}$
}

\author{
${ }^{a}$ Departamento de Engenharia de Materiais, Universidade Federal de Campina Grande, 58109-970, \\ Campina Grande, PB, Brasil \\ ${ }^{b}$ Instituto de Cerámica y Vidrio (ICV), Consejo Superior de Investigaciones Cientificas (CSIC), Calle \\ Kelsen, 5, 28049 Madrid, Spain
}

Received: February 08, 2018; Revised: April 19, 2018; Accepted: May 06, 2018

\begin{abstract}
The aim of this work was to perform an analysis of the influence of Sodium Fluoride (NaF) on chitosan particles size and morphology produced via ionotropic gelation with higher rotational stirring speed. Samples were characterized by Fourier Transform Infrared Spectroscopy (FTIR), Dynamic Light Scattering (DLS), Zeta Potential ( $\zeta$ ), Scanning Electron Microscopy and Energy Dispersive Spectroscopy (SEM/EDS). Chitosan/TPP particles diameter obtained was in the order of $3.8 \mu \mathrm{m}$. When $\mathrm{NaF}$ was added in different concentration $(0.05 \%, 0.2 \%$ and $2 \%)$, the particle size diameter values, Zeta Potential and Poly Dispersive Index measures consequently decreased. Even so, no further modification in morphology was found. An exception was made for the samples with higher $\mathrm{NaF}$ concentration. After 21 days, occurred an increase in the particle size diameter. In the future, the proposed methodology could provide a dentistry application, especially on delivering particles of $\mathrm{NaF}$.
\end{abstract}

Keywords: chitosan, fluoride, particles.

\section{Introduction}

Chitosan nanoparticles and chitosan microparticles have been investigated in the past few years due to potential applications on medical and pharmaceutical fields ${ }^{1}$ such as gene carriers, proteins ${ }^{2,3,4}$, drugs, cancer diagnosis and treatment ${ }^{5,6}$. To produce chitosan particles in several different sizes, ionotropic gelation method is generally applied. This method consists of a reaction of protonated chitosan with multivalent polyanions. Chitosan jellifies rapidly as a consequence of the formation of inter- and intramolecular bonds between the chitosan amino group and multivalent polyanions. The multivalent polyanions that have been widely used is sodium tripolyphosphate (TPP) because it is non-toxic and has a rapid gelling ability ${ }^{7,8,9,10}$ and also for being considered a substantial food additive by the U.S. Food and Drug Administration 6,11 .

For ionotropic gelation method, different factors could influence particle size and distribution. Chitosan polymeric chain, in the presence of an acid solution, is exposed to electrostatic repulsion and in turn, the polymeric chain flexibility is affected. Thus, an extended conformation could occur. Protonated amino groups from chitosan reacts with TPP ions, resulting in an ionotropic gelation reaction. Therefore, chitosan initial state, on the presence of acetic acid solution, has a significant effect on final product physical properties. While chitosan / TPP suspension is a metastable system, the ionic strength changed and the $\mathrm{pH}$ value drastically modified the aggregation of chitosan molecules ${ }^{6,12}$. In the previous study, a number of factors influencing this system has been proposed, such as solution concentration, chitosan / TPP ratio, $\mathrm{pH}$, molecular weight $\mathrm{t}^{9,13}$ and mixing conditions (mechanical stirring or sonification) ${ }^{14}$.

Recent studies reported that by adding moderate amounts of salt, such as $\mathrm{NaCl}^{6,9,15}$ it is possible to obtain a narrower particle size distribution. Huang e Lapitsky conducted a study in order to investigate the influence of sedimental parts of $\mathrm{NaCl}$ on the chitosan/TPP microgel yield. Researchers found that the presence of $\mathrm{NaCl}$ enhances the colloidal stability and a narrower particle size distribution was produced $^{15}$.

Jonassen and co-authors studied the concentration of chitosan nanoparticles obtained by ionotropic gelation with and without addition of $\mathrm{NaCl}$. The results showed the presence of the monovalent salt draw in smaller and more compact particles and it is possible to obtain particles in the nanometer range with higher polymer concentration ${ }^{6}$. Antoniiou evaluated the chitosan nanoparticles optimization via the ionotropic gelation method. The researcher found that diluting the $\mathrm{NaCl}$ solution in the Ionic composition decreases the particle size by $25 \%$, as well as a size distribution of narrow particle?.

Another type of monovalent salt can be used is NaF. The Sodium Fluorine $(\mathrm{NaF})$ is widely used in dentistry applications as an agent to control dental caries and can be released from glass ionomer cements, composite resin and toothpaste ${ }^{10,16}$. Even though, there are few applications to obtain these kind of particles with $\mathrm{NaF}$. 
Therefore, the aim of this work was to perform an analysis of the Sodium Fluoride (NaF) addition the influence on chitosan particles size and morphology produced via ionotropic gelation.

\section{Materials and Methods}

\subsection{Materials}

Medium molar mass Chitosan (150-390 KDa) with a degree of deacetylation of $75-85 \%$ and sodium tripolyphosphate (TPP) were purchased from Sigma Aldrich, Acetic Acid and Sodium Flourine $(\mathrm{NaF})$ were purchased from Vetec, and deionized water without further purification.

\subsection{Chitosan particle preparation $(C)$}

Chitosan particles were prepared following the ionotropic gelation method, reported previously ${ }^{17}$. Chitosan solution $(5 \mathrm{mg} / \mathrm{mL})$ was prepared via polymer powder dissolution with acetic acid solution $(0.14 \% \mathrm{v} / \mathrm{v})$ under magnetic stirring. TPP solution was prepared to obtain a final $2 \mathrm{mg} / \mathrm{mL}$ concentration. The final chitosan solution was dispersed in a high-speed dispersant (IKA ultra-turrax T25 digital) at 20.000 RPM and simultaneously, TPP solution was dropped in. The final mixture was placed under higher rotation speed for 10 minutes. Then, the solution was centrifuged for 30 minutes at 3.500 RPM.

\subsection{Chitosan/NaF particles preparation ( $\mathrm{CNaF}$ )}

Chitosan solution $(5 \mathrm{mg} / \mathrm{mL})$ was prepared following the same procedure from item 2.2. After the dissolution step, $\mathrm{NaF}$ solution was added in different concentrations $(0.05 \%, 0.2 \%$ and $2 \% \mathrm{~m} / \mathrm{v}$ ) with $900 \mathrm{ppm}$ of $\mathrm{F}$ and kept under mechanical stirring for 30 minutes. After homogenization, TPP solution was dropped and the final mixture was placed under high rotation for 10 minutes. Then, the solution was centrifuged for 30 minutes at 3.500 RPM, and called of $\mathrm{CNaF} 0.05, \mathrm{CNaF} 0.2$ and $\mathrm{CNaF} 2$, respectively for $0.05 \%, 0.02 \%$ and $2 \% \mathrm{NaF}$.

\subsection{Particles characterization}

\subsubsection{Fourier transform infrared spectroscopy (FTIR)}

The samples were poured into Petri dishes (90 cm X 15 $\mathrm{cm})$, frozen in a freezer $\left(-18^{\circ} \mathrm{C}\right)$ for $48 \mathrm{~h}$ and lyophilized for another $48 \mathrm{~h}$. After this step, the characterization by FTIR was carried out in order to identify the functional groups that correspond to the energy levels of the molecule of the chitosan nanoparticles. A spectrometer Spectrum 400, FT-IR / FT-NIR Spectrometer Perkin Elmer, with a resolution of $4 \mathrm{~cm}^{-1}$ and 20 sweeps, and with a scan of 4000 to $650 \mathrm{~cm}^{-1}$ was used, with ATR (attenuated total reflectance) device.

\subsubsection{Size particles measures}

The Zetapals - Brookhaven equipment was applied to measure the Dynamic Light Scattering - DLS and the Poly Dispersive Index (PDI). The BI-SCP cell was used at 25 ${ }^{\circ} \mathrm{C}$ with three parameters: chitosan refractive index (IR) of 1.700, absorption index 0.010 and water solvent RI: 1.33 , with a viscosity of $0.8872 \mathrm{cP}$. All the measures were performed in triplicates.

Samples were stored on falcon tubes in a refrigerator for $24 \mathrm{~h}$ and after this period, was conducted the DLS analysis.

\subsubsection{Zeta potential (ל)}

The Zetapals - Brookhaven equipment was applied to measure the Zeta Potential $(\zeta)$. The BI-SCP cell was used at $25{ }^{\circ} \mathrm{C}$ with three parameters: chitosan refractive index (IR) of 1.700, absorption index 0.010 and for water solvent RI: 1.33 , with a viscosity of $0.8872 \mathrm{cP}$. The Zeta Potential $(\zeta)$ measures was conducted with the same equipment, but with the following electrode AQ-1214. All the measurements were performed in triplicates.

Samples were stored on falcon tubes in a refrigerator for $24 \mathrm{~h}$ and after this period, the zeta potential analysis was conducted.

\subsubsection{Scanning electron microscopy (SEM)}

SEM was applied to evaluate the size and morphology of the produced particles. The samples were dried in a silica desiccator for $24 \mathrm{~h}$. After this period, the samples were submitted to a gold thin layer for 3 minutes in an electrical current of $10 \mathrm{~mA}$. SEM Phenom Pro-X with depth of focus of $1 \mathrm{~mm}$, with resolution of $30 \mathrm{~nm}$ and electrical voltage of $15 \mathrm{kV}$, was used to analyze the samples, under low vacuum and random pressure (1 to $270 \mathrm{~Pa}$ ). To identify chemical elements, Energy dispersive spectroscopy (EDS) coupled accessory was used.

Samples were stored on falcon tubes in a refrigerator and after 21 days and analyzed by MEV and EDS.

To measure the particles diameter, the ImageJ software program was used in previous selected micrographs $(\mathrm{C}$, $\mathrm{CNaF} 0.05, \mathrm{CNaF} 0.2$ and $\mathrm{CNaF} 2$ ).

\section{Results and Discussion}

\subsection{Fourier transform infrared spectroscopy (FTIR)}

Figure 1 showed the FTIR spectra of TPP, Chitosan Solution (CS), Chitosan Particles (C) and Chitosan Particle with Sodium Fluorine (CNaF0.05, CNaF0.2 and $\mathrm{CNaF} 2)$. 


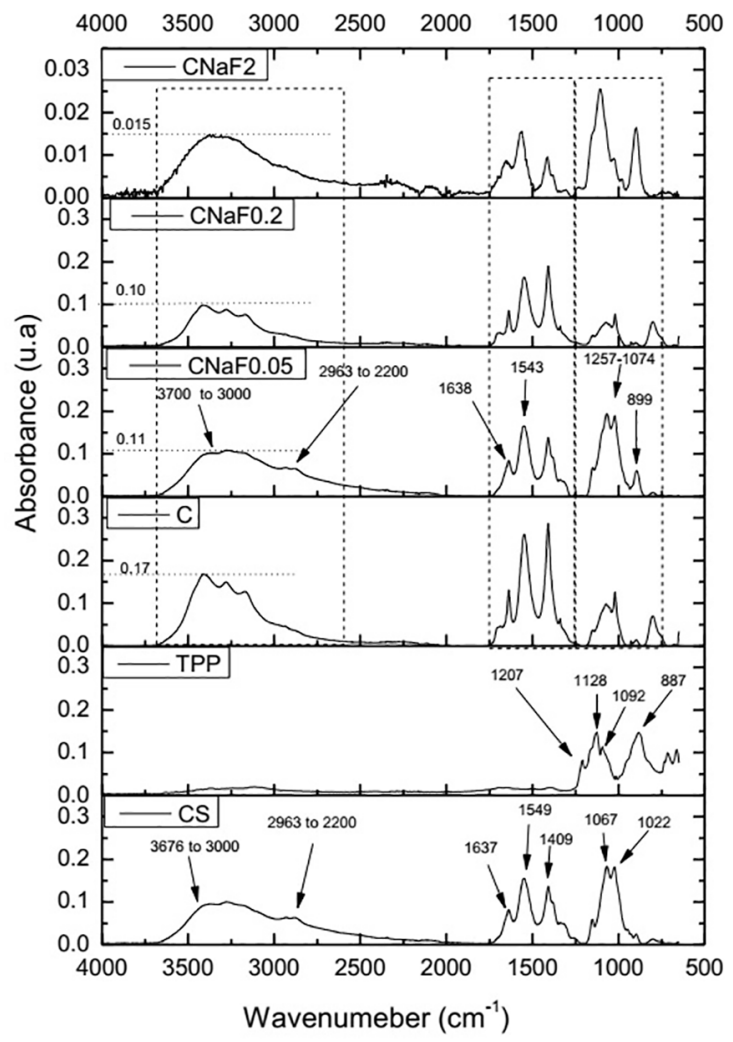

Figure 1. FTIR spectra of: TPP, Chitosan Solution (CS), Chitosan Particles (C), CNaF0.05, CNaF0.2 and $\mathrm{CNaF} 2$, respectively for $0.05 \%, 0.02 \%$ and $2 \% \mathrm{NaF}$.

The FTIR spectra detected one vibration at $1207 \mathrm{~cm}^{-1}$ to $\mathrm{P}=\mathrm{O}$ stretching. Symmetrical and asymmetrical vibration located at $1128 \mathrm{~cm}^{-1}$ and $1092 \mathrm{~cm}^{-1}$, are attributed to the stretching of the $\mathrm{PO}_{2}$ groups and the $\mathrm{PO}_{3}$ groups, respectively. Finally, at $887 \mathrm{~cm}^{-1}$, is attributed to the asymmetrical stretching of the P-O-P bond'.

The CS FTIR spectra shows absorption at 3676 to 3000 $\mathrm{cm}^{-1}$ typical from chitosan stretching vibrations. At 2963 to $2200 \mathrm{~cm}^{-1}$ is related to an overlap in stretching for functional $\mathrm{OH}$ and $\mathrm{NH}_{2}$ groups. All this absorption is typical from hydrogen bonds formation. The bands located at $1637 \mathrm{~cm}^{-1}$ and in $1549 \mathrm{~cm}^{-1}$ are attributed to the absorption of $\mathrm{C}=\mathrm{O}$, relative to the stretching of carbonyl groups $\left(-\mathrm{NHCOCH}_{3}\right)$ and to the amine group $\left(\mathrm{NH}_{2}\right)$, respectively, once the chitosan is not fully deacetylated. At $1409 \mathrm{~cm}^{-1}$ is related to the vibration of the deformation of amino group (N-H). The band located in $1067 \mathrm{~cm}^{-1}$ is attributed to the primary alcohol and finally, the bands located in 1155,1022 and $901 \mathrm{~cm}^{-1}$ are attributed to C-O-C stretching, respectively9.

Concerning the spectra of $\mathrm{C}, \mathrm{CNaF} 0.05, \mathrm{CNaF} 0.2$ and $\mathrm{CNaF} 2$ samples, bands values between $3700 \mathrm{~cm}^{-1}$ and 3000 $\mathrm{cm}^{-1}$, are associated to $\mathrm{O}-\mathrm{H}$ and $\mathrm{N}-\mathrm{H}$ stretching vibration. Concerning the band values between $2999 \mathrm{~cm}^{-1}$ and 2969 $\mathrm{cm}^{-1}$ are attributed to the overlapping of stretching of $\mathrm{OH}$ and $\mathrm{NH}_{2}$ groups, which belongs to chitosan. In this same region, modifications could be identified, which are perceived by the hydrogen bond intensities reduction. Still, this reduction refers to the $-\mathrm{NH}_{2}$ groups protonation and a broader elongation of protonated amine group $\left(-\mathrm{NH}_{3}^{+}\right)$vibrations. Also, this reduction could be assigned to the presence of TPP crosslink bonds on chitosan crystalline structure. Between 1257-1074 $\mathrm{cm}^{-1}$ a reduction is related to the overlapping of $\mathrm{P}=\mathrm{O}$ group from TPP, to the primary alcohol $\left(1062 \mathrm{~cm}^{-1}\right)(\mathrm{OH})$ and C-O-C stretching $\left(1027 \mathrm{~cm}^{-1}\right)$. The band at $899 \mathrm{~cm}^{-1}$ has a relationship to P-O with P-O-P, likewise as a result of the TPP crosslinked on chitosan molecules. The $1638 \mathrm{~cm}^{-1}$ region corresponds to amide $\left(\mathrm{NH}_{2} \mathrm{CH}_{3}\right)$, and in $1543 \mathrm{~cm}^{-1}$ correspond to amino group $\left(\mathrm{NH}_{2}\right)$.

Comparing the CS spectra with the $\mathrm{C}$ samples obtained, it is possible to verify changes in the $\mathrm{C}$ spectrum, indicating an interaction between the amino groups of chitosan protonated with the phosphate group of TPP. This result is supported by the research of Antoniou and Anitha ${ }^{1,9}$.

Anitha and her co-workers studied the development of mucoadhesive thiolated chitosan nanoparticles for biomedical applications. They obtained chitosan nanoparticles by ionotropic gelation using TPP as a crosslinking agent. The results showed characteristic from chitosan bands and modifications in the regions of $1637 \mathrm{~cm}^{-1}$ indicating the crosslinking reaction between chitosan and TPP .

Antoniou and co-workers also investigated the chitosantripolyphosphate nanoparticles obtaining by ionotropic gelation method. The results showed changes in the region of 3421 $\mathrm{cm}^{-1}, 1568 \mathrm{~cm}^{-1}$ and $1630 \mathrm{~cm}^{-1}$ indicating the interaction between the amino group of chitosan and TPP'.

The addition $\mathrm{NaF}$ reflected in a reduction in intensity at $1547 \mathrm{~cm}^{-1}$. Studies carried out by Huang investigated the influence of $\mathrm{NaCl}$ addition on chitosan/TPP microgel. As a reflection of the addition of moderate amounts of $\mathrm{NaCl}$, a decrease in the binding strength on chitosan/TPP system was detected. Also turn them down as a result of competition between TPP and $\mathrm{NaCl}$. When reducing the binding rate, compared to absence of $\mathrm{NaCl}$, probably resulting in a homogeneous distribution of TPP bound to chitosan ${ }^{15}$.

\subsection{Size particle determination, poly dispersive index (PDI) and zeta potential ( ()}

Table 1 shows the data obtained from mean particle diameter, PDI, Zeta potential and $\mathrm{pH}$ after $24 \mathrm{~h}$ that samples preparation.

In Table 1, the chitosan particle diameter was $3.869 \mu \mathrm{m}$ and when sodium fluoride was added, a particle diameter reduction was detected for $\mathrm{CNaF0} 0.05, \mathrm{CNaF} 0.2$ and $\mathrm{CNaF}$, to $1.083 \mu \mathrm{m}, 0.731 \mu \mathrm{m}$ and $0.679 \mu \mathrm{m}$, respectively. The presence of $\mathrm{NaF}$ made it possible to obtain particles in nanometric range; similar results were also obtained by ${ }^{9,10,15}$. This result is already expected once chitosan is solubilized; protonated chitosan amino groups extended and prolonged the chain conformation as a result of an electrostatic repulsion. 
Tabel 1. Mean diameter of the particles, PDI and zeta potential for, $\mathrm{CS}, \mathrm{C}, \mathrm{CNaF} 0.05, \mathrm{CNaF} 0.2$ and $\mathrm{CNaF}$.

\begin{tabular}{lcccc}
\hline Samples & Particle Diameter $(\mu \mathrm{m})$ & PDI & Zeta Potential $(\mathrm{mV})$ & $\mathrm{pH}$ \\
$\mathrm{CS}$ & - & - & $50.47( \pm 0.75)$ & 5.0 \\
$\mathrm{C}$ & $3.869( \pm 0.192)$ & $4.8( \pm 1.17)$ & $38.49( \pm 0.76)$ & 5.4 \\
$\mathrm{CNaF} 0.05$ & $1.083( \pm 0.007)$ & $0.33( \pm 0.12)$ & $26.78( \pm 0.55)$ & 5.5 \\
$\mathrm{CNaF} 0.2$ & $0.731( \pm 0.120)$ & $0.9( \pm 0.31)$ & $17.79( \pm 1.16)$ & 5.6 \\
$\mathrm{CNaF} 2$ & $0.679( \pm 0.079)$ & $0.6( \pm 0.26)$ & $4.83( \pm 0.84)$ & 6.2 \\
\hline
\end{tabular}

Also, TPP in chitosan solution cause crosslinking bonds and promotes the formation of larger particles and particle aggregates, as reported in the literature ${ }^{6,15}$. However, in the presence of $\mathrm{NaF}$, where fluorine $\left(\mathrm{F}^{-}\right)$can bind to $\mathrm{NH}_{3}^{+}$, a reduction on protonated amino group occurs, reducing the tendency of chitosan crosslinking, resulting in a more compact structure because of the reduction of electrostatic repulsion and screening of charges, and consequently, smaller particle sizes as shown in Table $1^{6}$.

The NaF concentration is inversely proportional to the PDI results (Table 1), following the same results shown in the particle size research results from ${ }^{9,15,10}$. This behavior is related to the fluorine chemical character (electronegative and reactive element). This behavior allows the connection with another types of ionic character atoms ${ }^{10}$, suggesting that the particle structural rearrangement plays a partial influence on the PDI ${ }^{15}$.

Regarding the results of zeta potential, as observed in Table 1, it is possible to identify that the CS presents positive zeta potential, since solubilized in acetic acid presents the amino group protonated as already reported in the literature ${ }^{6,9,15}$, when adding the TPP there is a reduction in the zeta potential, because the TPP occupies some of the positive charge of chitosan $\left(\mathrm{NH}_{3}^{+}\right)$due to crosslinking ${ }^{6}$ corroborating with the studies presented by ${ }^{6,15}$. By adding $\mathrm{NaF}$ in moderate proportions ( $\mathrm{CNaF} 0.05)$ there is a significant reduction of $31.2 \%$ in zeta potential, and by increasing these $\mathrm{NaF}$ ratios, there is an even greater reduction, $53.8 \%$ $(\mathrm{CNaF} 0.2)$ and $87.4 \%(\mathrm{CNaF} 2)$. This can be attributed to the presence of $\mathrm{NaF}$, since it was added before the crosslinker reducing the number of positive charges of chitosan, as the $\mathrm{NaF}$ concentration was increased, this reduction was even greater, corroborating with the studies carried out by $y^{6,9,15}$. It is also possible to identify that as the zeta potential reduces the $\mathrm{pH}$ of the system it gradually increases to 6.2 , suggesting the reduction of these positive charges of the chitosan presented at the zeta potential. Once the $\mathrm{pH}$ reaches values of 6.2, chitosan $\mathrm{pKa}$ values are achieve and thus, decreasing the dissolution capacity of the polymer, which could provide agglomerates formation ${ }^{2}$.

Huang and Lapitsky investigated the influence of $\mathrm{NaCl}$ addition on chitosan/TPP microgel production. Concerning this addition, resulting in a particle size reduction under equal conditions studied. Adding moderate amounts of $\mathrm{NaCl}$ has a directly impact on particle size and particle size distribution.
A smaller particle size distribution, PDI and zeta potential decrease was reported ${ }^{15}$.

Antoniou and co-workers also investigated the addition of $\mathrm{NaCl}$ in the Ionic composition, prior to obtaining chitosan nanoparticles by ionotropic gelation method. These results showed a narrow particle size distribution and a reduction in particle size in $25 \%$ as a result of change in Ionic strength in the chitosan chain. Along the ionotropic gelation process, the loading of the protonated amine group $\left(\mathrm{NH}_{3}^{+}\right)$is reduced to produce smaller sized particles. With the increase on the $\mathrm{NaCl}$ concentration, no significant changes $(\mathrm{P}>0.05)$ were observed in the particles PDI. For Zeta Potential there was a decrease due to the reduction of the interactions between chitosan and TPP9.

Nguyen and coworkers prepared chitosan nanoparticles in $0.2-0.4 \%(\mathrm{w} / \mathrm{w}) \mathrm{NaF}$, respectively $(0.2 \%$ and $0.4 \%)$ by the ionotropic gelation method and obtained a particle size of $101 \mathrm{~nm}$ and $112 \mathrm{~nm}$ and a low polydispersion index of 0.16 and 0.14 for the concentrations of $\mathrm{NaF} 0.2 \%$ and $0.4 \%$ respectively. Once again, these results are related to the Fluorine chemical character (electronegative and reactive element) which allows the connection with another types of Ionic character atoms. Nevertheless, chitosan polymeric chains enhance the TPP action as a crosslinking agent, resulting in a smaller and compacted nanoparticle scale matrix ${ }^{10}$.

Jonassen and coworkes studied the chitosan nanoparticles concentration produced via ionotropic gelation with and without addition of $\mathrm{NaCl}$. As result, the monovalent salt induced a positive and low zeta potential could be related to the presence of the monovalent salt screening of charges, as fill in some chitosan positive charges before reaction with $\mathrm{TPP}^{6}$.

\subsubsection{Scanning electron microscopy (SEM)}

Morphological properties and surface of $\mathrm{C}, \mathrm{CNaF} 0.05$, $\mathrm{CNaF} 0.2$ and $\mathrm{CNaF} 2$ samples, after 21 days, are shown in Figure 2.

Analyzing the micrograph Figure 2 (a) it is possible to identify that the obtained particles are agglomerated, has smooth surface and spherical shape. This was already an expected result since it was widely reported in literature ${ }^{4,18,19,20,21}$. Figure 2 (b), (c) and (d) show agglomerated particles with smoothie surface, spherical shape, and with NaF addition, no modification on the particle surface was detected. Also no formation of $\mathrm{NaF}$ crystals on the surface of the particle, corroborating with the data obtained by ${ }^{15,22}$. 

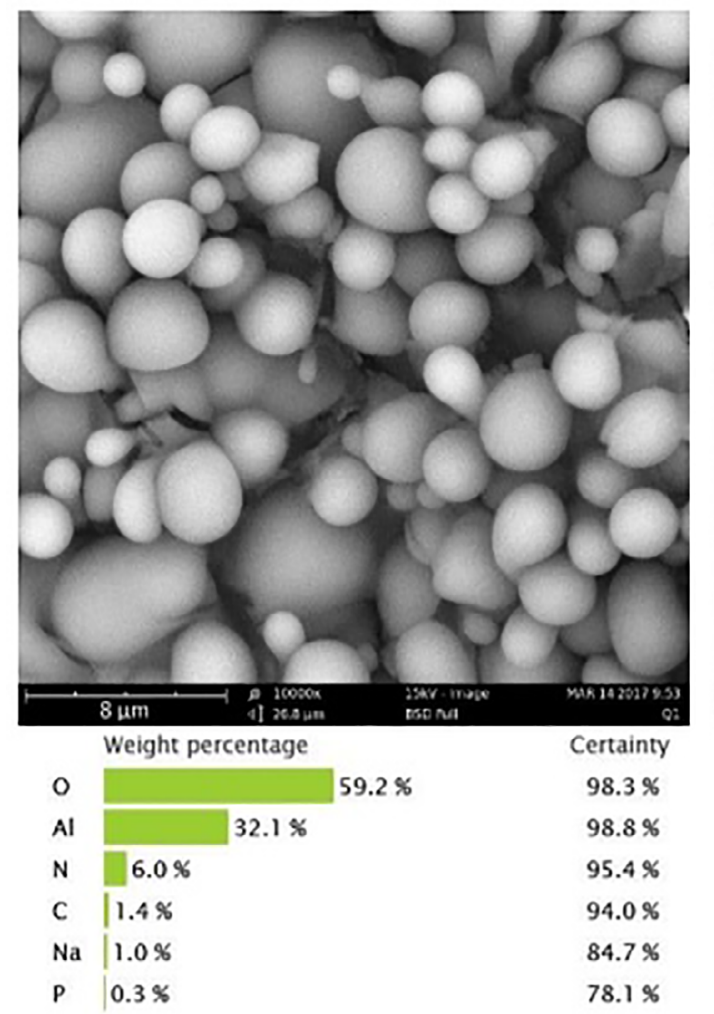

(a)

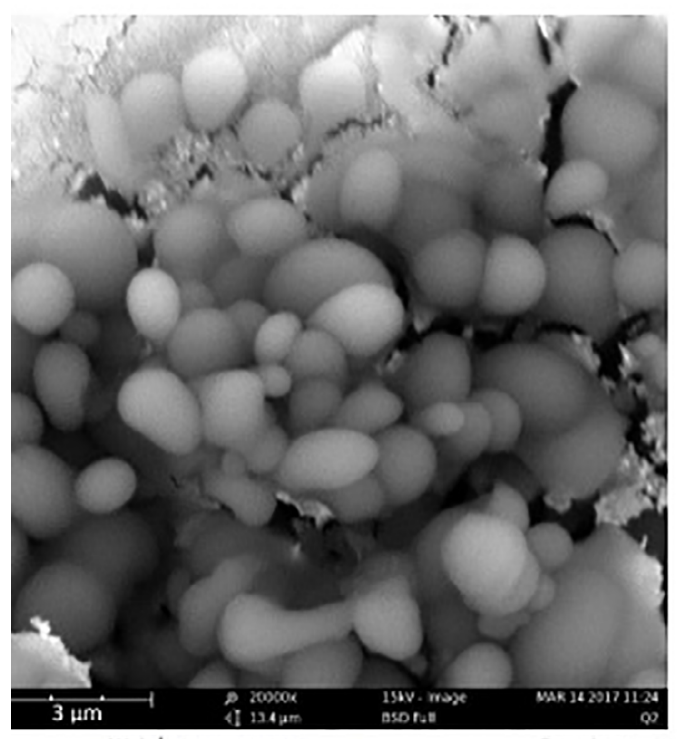

\begin{tabular}{|c|c|c|c|}
\hline & Weight percenta & & Certainty \\
\hline Al & & $69.1 \%$ & $99.3 \%$ \\
\hline 0 & $22.0 \%$ & & $96.6 \%$ \\
\hline $\mathrm{N}$ & $3.9 \%$ & & $91.0 \%$ \\
\hline $\mathrm{Na}$ & $1.7 \%$ & & $90.6 \%$ \\
\hline $\mathbf{F}$ & $1.3 \%$ & & $77.3 \%$ \\
\hline C & $1.0 \%$ & & $88.4 \%$ \\
\hline $\mathbf{p}$ & $0.9 \%$ & & $85.5 \%$ \\
\hline
\end{tabular}

(c)

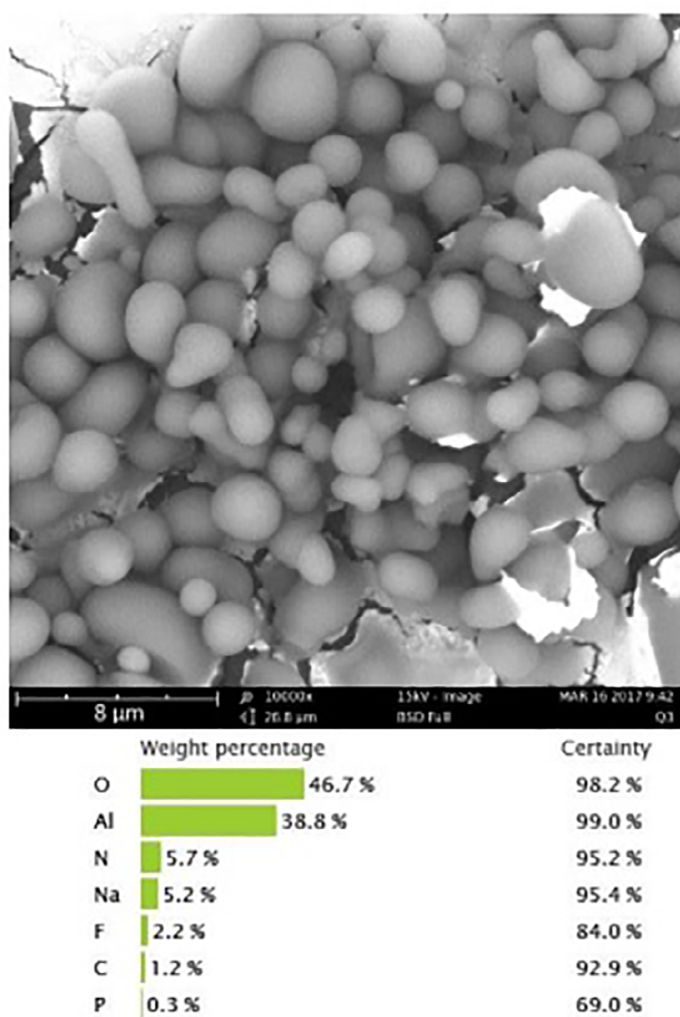

(b)

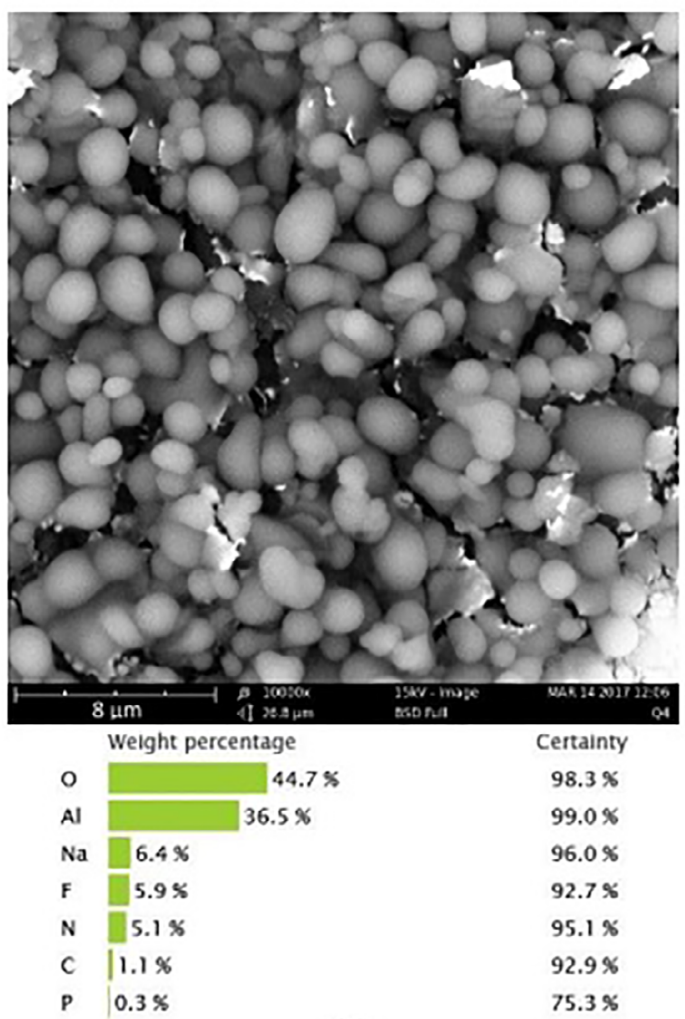

(d)

Figure 2. Particles micrographs / EDS (a) C, (b) CNaF0.05 e (d) CNaF2 10.000X, and (c) CNaF0.2 20.000X. 
In Figure 2 it is confirmed the presence of carbon, oxygen and nitrogen, three chitosan characteristic elements. The detection of sodium and phosphorus elements are attributed to TPP salt for both samples. The detection of aluminum is referred to the sample port. For the sample $\mathrm{CNaF} 0.05$, $\mathrm{CNaF} 0.2$ and $\mathrm{CNaF} 2$ the presence of fluorine is also confirmed.

On the other hand, only $\mathrm{CNaF} 2$ presented divergent regions when compared with other samples with $\mathrm{NaF}$

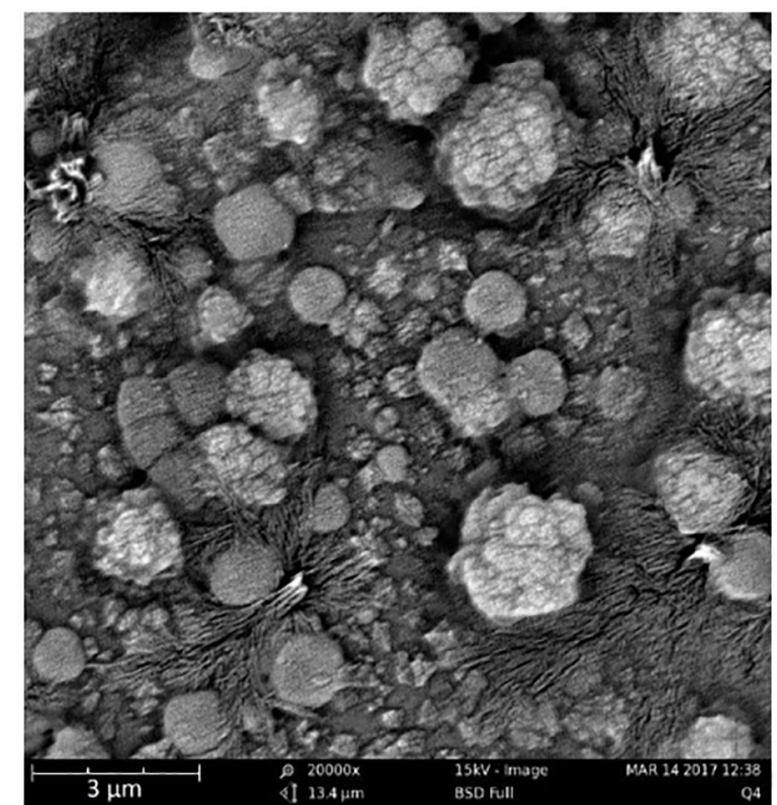

(Figure 3). The particles maintained the spherical shape, although with rough surfaces and a higher $F$ percentage when compared to the other regions (Figure 2(b), (c) and (d)). As this sample holds a greater amount of $\mathrm{NaF}$, it could be related to an excess on the particles, or a $\mathrm{F}^{-}$migrated effect or accumulated excess.

Figure 4 shows the histogram of the particle diameter obtained from Figure 2.

Figure 3. Particles micrographs / EDS CNaF2 20.000X.

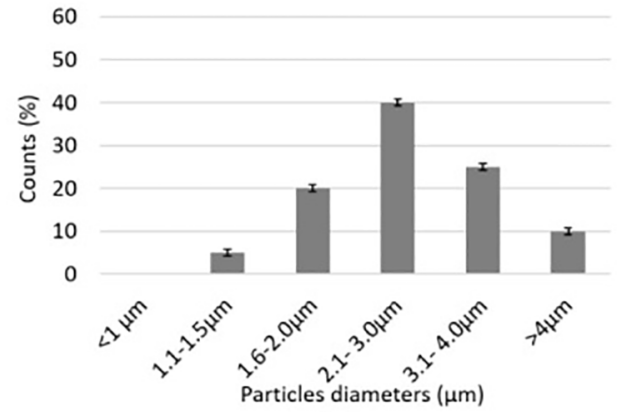

(a)

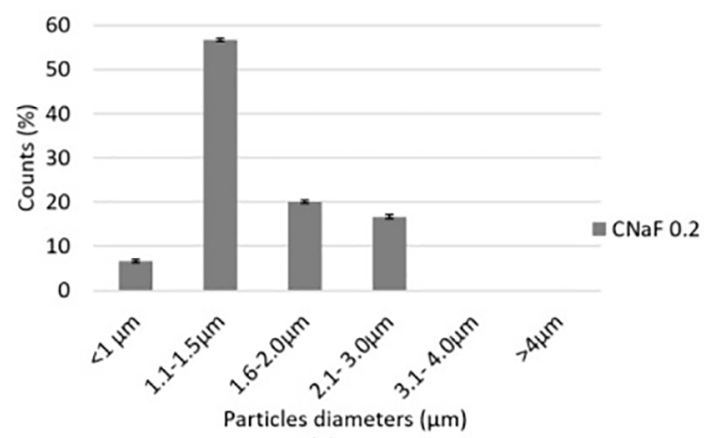

(c)

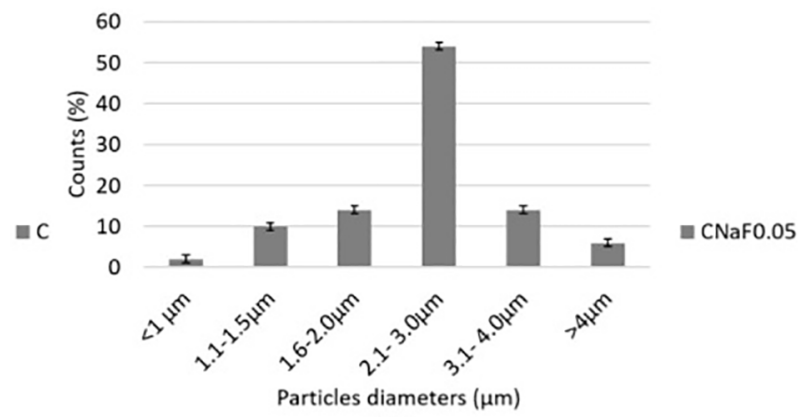

(b)

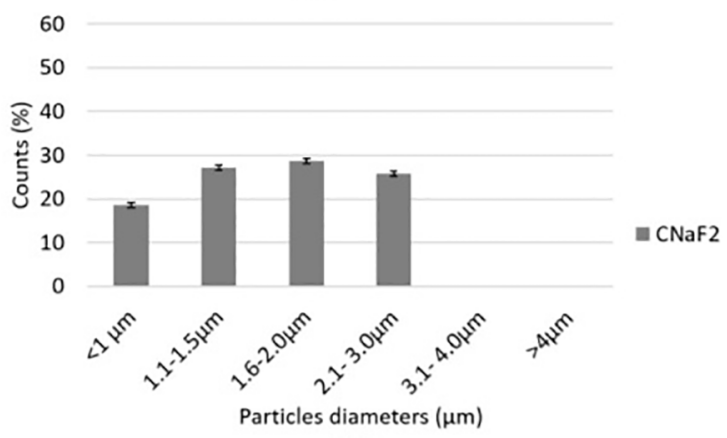

(d)

Figure 4. Particle diameter histogram $\mathrm{C}(\mathrm{a}), \mathrm{CNaF} 0.05$ (b), $\mathrm{CNaF0} 0.2$ (c) and $\mathrm{CNaF} 2$ (d). 
When analyzing Figure 4, it can be seen that the average diameter obtained by the micrograph of samples $\mathrm{C}$ was 2.78um. However, it has a smaller particle diameter than that shown in Table 1, and this can be attributed to the volumetric retraction $(23 \%)$ of the particles, since the DLS analysis were performed with the swollen particles ${ }^{23,24}$.

A research conducted by Sotelo-Boyás and co-workers obtained chitosan nanoparticles and chitosan nanocapsules by nanoprecipitation method and embedded with lemon essential oil. From DLS results, nanoparticle diameters range from 6-18nm and from 60-900nm. On the other hand, TEM results showed nanoparticles diameter of $5.8 \mathrm{~nm}$. This difference in nanoparticles diameter range is a result of sample preparation once for TEM analysis, samples were dehydrated in ethanol or methanol and dried. Otherwise, in DLS analysis samples are in solution ${ }^{24}$. A research conducted by Luque-Alcaraz and co-workers presented similar behavior when chitosan nanoparticles showed DLS measure size results of $494 \mathrm{~nm}$ and SEM results for nanoparticles diameter range from $20-100 \mathrm{~nm}^{23}$.

Figure 4(b), (c) and (d) contain the particle diameter histogram and it is possible to observe average diameters of $2.67 \mu \mathrm{m}, 1.61 \mu \mathrm{m}$ and $1.68 \mu \mathrm{m}$ respectively. Compared with the sample $\mathrm{C}$ average diameter measurement $(2.78 \mu \mathrm{m})$, particles mentioned showed smaller diameter values.

During the 21-day period it is possible to observe, in Figure 3(a) that, there was no significant coalescence of the particles and this can be attributed to $\mathrm{pH} 5.0$ and zeta potential of $38.49 \mathrm{mV}$ (Table 1) resulting in a more stable system, since particles with zeta potential above $\pm 30 \mathrm{mV}$ are considered stable ${ }^{25}$. However, it is possible to observe in Figure 3 (b, cand d) that the presence of NaF did not prevent the particles coalescing after the period of 21 days, different from the results of Nguyen and colleagues ${ }^{10}$, but corroborating with the studies ${ }^{22}$. This can be attributed to the increase in $\mathrm{pH}$ and the zeta potential reduction, with increasing $\mathrm{NaF}$ concentration, as shown in Table 1, resulting in an unstable system, corroborating with the data obtained $\mathrm{by}^{2,22}$ that with increasing $\mathrm{pH}$ and reduction of zeta potential favors the formation of aggregates.

Nguyen and co-workers conducted research with chitosan/TPP nanoparticles embedded with $\mathrm{NaF}(0.2 \%$ and $0.4 \%$ ). The nanoparticles were produced, via the ionotropic gelation method, and stored for 30 days in a refrigerator to evaluate stability. Characterization via DLS were carried out to read the results of particle size and PDI. The results showed no significant changes in particle size and PDI for both studied samples ${ }^{10}$.

Studies conducted by Liu and co-workers evaluated the stability and $\mathrm{pH}$ influence on chitosan nanoparticles obtained via water-oil emulsion method and with glutaraldehyde as a crosslinking agent with $\mathrm{NaF}$. The results showed that with greater $\mathrm{pH}$, the increase in the average diameter occurred as a result of the decrease in repulsion between the particles, resulting in the aggregation of particles. Samples were stored at $4{ }^{\circ} \mathrm{C}$ to check the stability. After 20 days, samples were characterized by DLS. The DLS results showed that the particles had a duplicate size and after 251 days, the sizes were maintained. These results demonstrated that chitosan nanoparticles are stable at neutral $\mathrm{pH}$ and low temperatures $^{22}$.

In view of the presented results, it is possible to identify that the high speed of rotation (20.000 RPM) that was used in the preparation of all the samples, it is possible to suggest that it did not modify the morphology and particle size, although it needs more specific studies.

Tsai and co-workers evaluated the mean diameter of chitosan nanoparticles obtained by the ionotropic gelation method using two types of mixing conditions: ultrasonic radiation and mechanical shearing. The results indicated that with the increase of the ultrasonic (10,30 and 50) watts and mechanical speed (60, 300 and 1000) RPM there was the reduction of the particle size and that with the increase of the temperature of $25^{\circ} \mathrm{C}$ to $45^{\circ} \mathrm{C}$ for the ultrasound there was a reduction of the particle size, but for mechanical agitation it remained practically constant. Once chitosan is exposed to ultrasound or mechanical shearing, the polymeric chains could be fragmented at half or less compared with the original size, resulting in different particle sizes ${ }^{14}$.

\section{Conclusion}

From the studies performed, with the increase of the amount of $\mathrm{NaF}$ added, it was possible to obtain chitosan particles at the nano scale without modifying their morphology, except for the sample $\mathrm{CNaF} 2$. Therefore, the $\mathrm{NaF}$ addiction modified the chitosan particles properties produced by ionotropic gelation with TPP, significantly reduced the zeta potential and turn the $\mathrm{pH}$ near the neutral, favoring the aggregates formation. The high speed of rotation that was used in the preparation of all the samples did not modify the particle size. After the period of 21 days stored under refrigeration, the samples with $\mathrm{NaF}$ increased their diameter, resulting in an unstable system. Therefore, it was possible to achieve the production of chitosan particles according to the proposed methodology and the presence $\mathrm{NaF}$ made possible to obtain particles in nanometric range and could provide, in the future, a dentistry application, especially on delivering particles of $\mathrm{NaF}$.

\section{Acknowledgments}

The financial support from Coordenação de Aperfeiçoamento de Pessoal de Nível Superior/CAPES and Brazilian Ministry of Health are gratefully acknowledged. 


\section{References}

1. Anitha A, Deepa N, Chennazhi KP, Nair SV, Tamura H, Jayakumar R. Development of mucoadhesive thiolated chitosan nanoparticles for biomedical applications. Carbohydrate Polymers. 2011;83(1):66-73.

2. Mattu C, Li R, Ciardelli G. Chitosan nanoparticles as therapeutic protein nanocarriers: The effect of $\mathrm{pH}$ on particle formation and encapsulation efficiency. Polymer Composites. 2013;34(9):15381545 .

3. Shukla SK, Mishra AK, Arotiba OA, Mamba BB. Chitosanbased nanomaterials: A state-of-the-art review. International Journal of Biological Macromolecules. 2013;59:46-58.

4. Grenha A, Seijo B, Remuñán-López C. Microencapsulated chitosan nanoparticles for lung protein delivery. European Journal of Pharmaceutical Sciences. 2005;25(4-5):427-437.

5. Duse L, Baghdan E, Pinnapireddy SR, Engelhardt KH, Jedelská $\mathrm{J}$, Schaefer J, et al. Preparation and Characterization of Curcumin Loaded Chitosan Nanoparticles for Photodynamic Therapy. Physica Status Solidi (A). 2017;1700709. Epub ahead of print.

6. Jonassen H, Kjøniksen AL, Hiorth M. Effects of ionic strength on the size and compactness of chitosan nanoparticles. Colloid Polym Science. 2012;290(10):919-929.

7. Gan Q, Wang T, Cochrane C, McCarron P. Modulation of surface charge, particle size and morphological properties of chitosan-TPP nanoparticles intended for gene delivery. Colloids Surfaces B: Biointerfaces. 2005;44(2-3):65-73.

8. Dash M, Chiellini F, Ottenbrite RM, Chiellini E. Chitosan-A versatile semi-synthetic polymer in biomedical applications. Progress in Polymer Science. 2011;36(8):981-1014.

9. Antoniou J, Liu F, Majeed H, Qi J, Yokoyama W, Zhong F. Physicochemical and morphological properties of size-controlled chitosan-tripolyphosphate nanoparticles. Colloids and Surfaces A: Physicochemical and Engineering Aspects. 2015;465:137-146.

10. Nguyen S, Escudero C, Sediqi N, Smistad G, Hiorth M. Fluoride loaded polymeric nanoparticles for dental delivery. European Journal of Pharmaceutical Sciences. 2017;104:326-334.

11. Lin YH, Sonaje K, Lin KM, Juang JH, Mi FL, Yang HW, et al. Multi-ion-crosslinked nanoparticles with $\mathrm{pH}$-responsive characteristics for oral delivery of protein drugs. Journal of Controlled Release. 2008;132(2):141-149.

12. Bao H, Li L, Zhang H. Influence of cetyltrimethylammonium bromide on physicochemical properties and microstructures of chitosan-TPP nanoparticles in aqueous solutions. Journal of Colloid and Interface Science. 2008;328(2):270-277.

13. Sipoli CC, Santana N, Shimojo AAM, Azzoni A, de la Torre LG. Scalable production of highly concentrated chitosan/TPP nanoparticles in different $\mathrm{pHs}$ and evaluation of the in vitro transfection efficiency. Biochemical Engineering Journal. 2015;94:65-73.
14. Tsai ML, Bai SW, Chen RH. Cavitation effects versus stretch effects resulted in different size and polydispersity of ionotropic gelation chitosan-sodium tripolyphosphate nanoparticle. Carbohydrate Polymers. 2008;71(3):448-457.

15. Huang Y, Lapitsky Y. Monovalent Salt Enhances Colloidal Stability during the Formation of Chitosan/Tripolyphosphate Microgels. Langmuir. 2011;27(17):10392-10399.

16. Wang Z, Shen Y, Haapasalo M. Dental materials with antibiofilm properties. Dental Materials. 2014;30(2):e1-16.

17. Calvo P, Remuñán-López C, Vila-Jato JL, Alonso MJ. Novel hydrophilic chitosan-polyethylene oxide nanoparticles as protein carriers. Journal of Applied Polymer Science. 1997;63(1):125-132.

18. Huang Y, Lapitsky Y. Salt-Assisted Mechanistic Analysis of Chitosan/Tripolyphosphate Micro- and Nanogel Formation. Biomacromolecules. 2012;13(11):3868-3876.

19. Khanmohammadi M, Elmizadeh H, Ghasemi K. Investigation of Size and Morphology of Chitosan Nanoparticles Used in Drug Delivery System Employing Chemometric Technique. Iranian Journal of Pharmaceutical Research. 2015;14(3):665-675.

20. Katas H, Raja MAG, Lam KL. Development of chitosan Nanoparticles as a Stable Drug Delivery System for Protein/ siRNA. International Journal of Biomaterials. 2013;2013:146320.

21. Mohammadpour DN, Eskandari R, Avadi MR, Zolfagharian H, Mir MSA, Rezayat M. Preparation and in vitro characterization of chitosan nanoparticles containing Mesobuthus eupeus scorpion venom as an antigen delivery system. Journal of Venomous Animals and Toxins including Tropical Diseases. 2012;18(1):44-52.

22. Liu H, Chen B, Mao Z, Gao C. Chitosan nanoparticles for loading of toothpaste actives and adhesion on tooth analogs. Journal of Applied Polymer Science. 2007;106(6):42484256.

23. Luque-Alcaraz AG, Cortez-Rocha MO, Velázquez-Contreras CA, Acosta-Silva AL, Santacruz-Ortega HDC, Burgos-Hernández A, et al. Enhanced Antifungal Effect of Chitosan/Pepper Tree (Schinus molle) Essential Oil Bionanocomposites on the Viability of Aspergillus parasiticus Spores. Journal of Nanomaterials. 2016;2016:6060137.

24. Sotelo-Boyás ME, Correa-Pacheco ZN, Bautista-Baños S, Corona-Rangel ML. Physicochemical characterization of chitosan nanoparticles and nanocapsules incorporated with lime essential oil and their antibacterial activity against food-borne pathogens. $L W T$. 2017;77:15-20.

25. Barreras US, Méndez FT, Martínez REM, Valencia CS, Rodríguez PRM, Rodríguez JPL. Chitosan nanoparticles enhance the antibacterial activity of chlorhexidine in collagen membranes used for periapical guided tissue regeneration. Materials Science and Engineering: C. 2016;58:1182-1187. 